\section{Berufsverband der Deutschen Urologen e.V.}

Urologe $2018 \cdot 57: 354$

https://doi.org/10.1007/s00120-018-0615-5

(c) Springer Medizin Verlag GmbH, ein Teil von Springer Nature 2018
Redaktion

W. Bühmann (V.i.S.d.P.)

Keitumer Süderstr. 33 c

25980 Sylt OT Keitum

\title{
$\#+4$ URO-Telegramm
}

\section{Gesundheitsausgaben betrugen erstmals mehr als eine Milliarde Euro pro Tag}

Wiesbaden - Die Gesundheitsausgaben haben im vergangenen Jahr in Deutschland die Marke von einer Milliarde Euro pro Tag überschritten. Das berichtete das Statistische Bundesamt heute in Wiesbaden auf der Basis einer eigenen Prognose. Die Ausgaben seien demnach im Vergleich $\mathrm{zu}$ 2016 um 4,9\% auf 374,2 Milliarden Euro angestiegen.

$\mathrm{Zu}$ den Gesundheitsausgaben zählen nach dieser Rechnung sämtliche Ausgaben für Güter und Dienstleistungen, die Staat, Privathaushalte und Unternehmen für Prävention, Behandlung, Nachsorge, Investitionen in Einrichtungen, zur Deckung von Verwaltungskosten oder für Transferleistungen ausgeben. Grundlage ist eine gemeinsame Definition, die von der Weltgesundheitsorganisation, der Organisation für wirtschaftliche Zusammenarbeit und Entwicklung sowie der europäischen Statistikbehörde Eurostat erarbeitet wurde.

\section{Mehrkosten durch drittes Pflegestärkungsgesetz}

2016 hatten sich die Gesundheitsausgaben nach dieser Ausgabenberechnung noch auf etwa 356,5 Milliarden Euro belaufen, was einem Anteil von 11,3\% am gesamten Bruttoinlandsprodukt entsprach. Damals hatte es im Vergleich zum Vorjahr einen Anstieg um 3,8\% gegeben.
Dass die Steigerung von 2016 auf 2017 höher war, lag laut Statistischem Bundesamt daran, dass im vorigen Jahr das dritte Pflegestärkungsgesetz in Kraft trat. Es erhöhte die finanziellen Leistungen für Pflegebedürftige und führte neue Ansprüche für Menschen mit Demenz ein.

2016 entfiel der größte Teil der Gesundheitsausgaben nach diesen Angaben auf die Sozialversicherungsbeiträge der Arbeitnehmer, die sich auf 112,4 Milliarden Euro beliefen. Es folgten die Sozialversicherungsbeiträge der Arbeitgeber (81,6 Milliarden) sowie die laut Definition enthaltenen staatlichen Transferleistungen (51,8 Milliarden Euro).

Dem Bundesamt zufolge werden Ausgaben für Forschung und Ausbildung sowie bestimmte staatliche Transferleistungen etwa zum Ausgleich krankheitsbedingter Folgen im Beruf nicht von der Definition erfasst. Daher wichen die Zahlen von Ausgabenrechnungen einzelner Sozialversicherungsträger $a b$. Das gelte insbesondere für Krankenkassen.

\section{(c) afp/aerzteblatt.de}

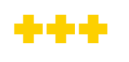

\section{Preise für Behandlungen stiegen im Krankenhaus überproportional}

Berlin - Die Preise für ärztliche Leistungen sind seit dem Jahr 2013 im Krankenhausbereich doppelt so stark angestiegen wie in den Arztpraxen. Das geht aus einer aktuellen Analyse des Zentralinstituts für die kassenärzt- liche Versorgung ( $\mathrm{Zi}$ ) hervor. Demnach erhöhten sich innerhalb von sechs Jahren die Preise im Krankenhaus um 15,8\%, im ambulanten Bereich um 7,3\%.

Der Grund für die unterschiedliche Entwicklung liegt dem $\mathrm{Zi}$ zufolge in den gesetzlichen Vorgaben. Nach Paragraf 10 Absatz 6 Krankenhausentgeltgesetz (KHEntgG) richtet sich die jährliche Veränderungsrate für den Basisfallwert grundsätzlich nach dem Anstieg der Kosten. Steigen die beitragspflichtigen Löhne und Gehälter der Versicherten stärker als die Kosten, wird diese höhere Rate an die Krankenhäuser weitergegeben (Meistbegünstigungsklausel).

\section{Verhandlung mit den Kassen}

Auch für die Leistungen der niedergelassenen Ärzte wird laut $\mathrm{Zi}$ jährlich ein Punktwert festgelegt. Dieser muss aber mit den Krankenkassen ausgehandelt werden. Es fehle jedoch eine entsprechende Regelung wie im Krankenhausentgeltgesetz, durch die sichergestellt würde, dass die Krankenkassen Erträge in wirtschaftlich guten Zeiten auch an die Praxen weitergeben.

Durch die Meistbegünstigungsklausel sollen insbesondere die Personalkosten in den Krankenhäusern finanziert werden, so das Zi. Die Personalkosten machen dem Institut zufolge etwa $66 \%$ aller Kosten eines Krankenhauses aus. Dabei stieg der Basisfallwert für die Krankenhäuser deutlich stärker als der Nominallohnindex des Statistischen Bundesamts, sodass im Vergleich zur allgemeinen Lohnentwicklung mehr Geld in die Krankenhäuser floss.

In den Arztpraxen liegt der Personalkostenanteil mit etwa
$75 \%$ deutlich höher als in den Krankenhäusern. Die Entwicklung des Orientierungswerts blieb aber ein Drittel hinter der allgemeinen Nominallohnentwicklung zurück.

„Im Ergebnis führt dies dazu, dass Arztpraxen immer weniger Wert werden“, erklärte Zi-Geschäftsführer Dominik von Stillfried. Im Vergleich zu den Krankenhäusern sei das eine einseitige und nicht nachvollziehbare Benachteiligung der Vertragsärzte. „Wer die Niederlassung fördern will, muss hier ansetzen“, so von Stillfried.

\section{(c) hil/sb/aerzteblatt.de}

